\title{
A mediastinal mass with abdominal and pulmonary presentation
}

\author{
Pranav Ish, Abanti Das, Nitesh Gupta, Juvva Kishan Srikanth, Sakshi Batra, Shibdas Chakrabarti
}

Vardhaman Mahavir Medical College \& Safdarjung Hospital, New Delhi, India

A 60-year-old male presented with the acute onset of breathlessness and dry cough. He had a documented weight loss of $5 \mathrm{kgs}$ with the lack of appetite. There was no history of fever, cough, sputum or haemoptysis. There was history of smoking with 50 pack-years and no history of alcohol intake. On examination, the patient had icterus. There was no palpable lymphadenopathy, clubbing or anasarca. On the respiratory system examination, right infrascapular air entry was reduced and hepatomegaly was present. Frontal chest radiograph (Figure 1A) and contrast enhanced CT (CECT) chest were done (Figure $1 \mathrm{~B}-\mathrm{D}$ ).

Considering the history and CT findings of a vertically oriented, fusiform-shaped posterior mediastinal mass extending to the both sides, partially encasing aorta and the oesophagus with areas of coarse calcifications with no vertebral erosion or extension to intervertebral neural foramen, a differential diagnosis of inflammatory pseudotumour, neurogenic tumour and lung adenocarcinoma were kept.

The patient underwent ultrasound-guided corebiopsy which revealed a poorly differentiated malignant tumour with tumour cells arranged in diffuse sheets (Figure $1 \mathrm{E}-\mathrm{F}$ ).

The slides were subjected to immunohistochemistry stains. The tumour cells were positive for Vimentin and CD34 and negative for S-100, SMA, Desmin, Calretinin, TTF-1, EMA, Bcl-2 (Figure 1 G-I). Thus, a final diagnosis of spindle-cell sarcoma was made.

Due to limited published literature, spindle-cell sarcoma treatment guidelines remain unexplored [1]. Treatment options include surgery, chemotherapy and radiotherapy. Surgery has shown a modest improvement in mortality and hence is the first line therapy [1]. The use of adjuvant radiotherapy for SCS remains controversial, and the sensitivity of SCS to chemotherapy in the metastatic setting is highly variable [2]. Chemotherapeutic agents are also multiple - doxorubicin for metastatic spindle-cell carcinoma, dacarbazine, gemcitabine and docetaxel being the other agents used [3].

Our patient had poor performance status with severe airflow obstruction on spirometry. He was advised metered dose inhalers; nutritional buildup and counselled for chemotherapy. However, the man succumbed to his illness before any specific therapy could be initiated.

Thus, a high index of suspicion must be kept for uncommon malignancies, primarily sarcomas such as spindle-cell sarcoma as a differential for large mediastinal masses extending to multiple compartments without any specific imaging features. Diagnosis needs aggressive interdepartmental collaboration with invasive tissue sampling and advanced immunohistochemistry for making an early diagnosis. 

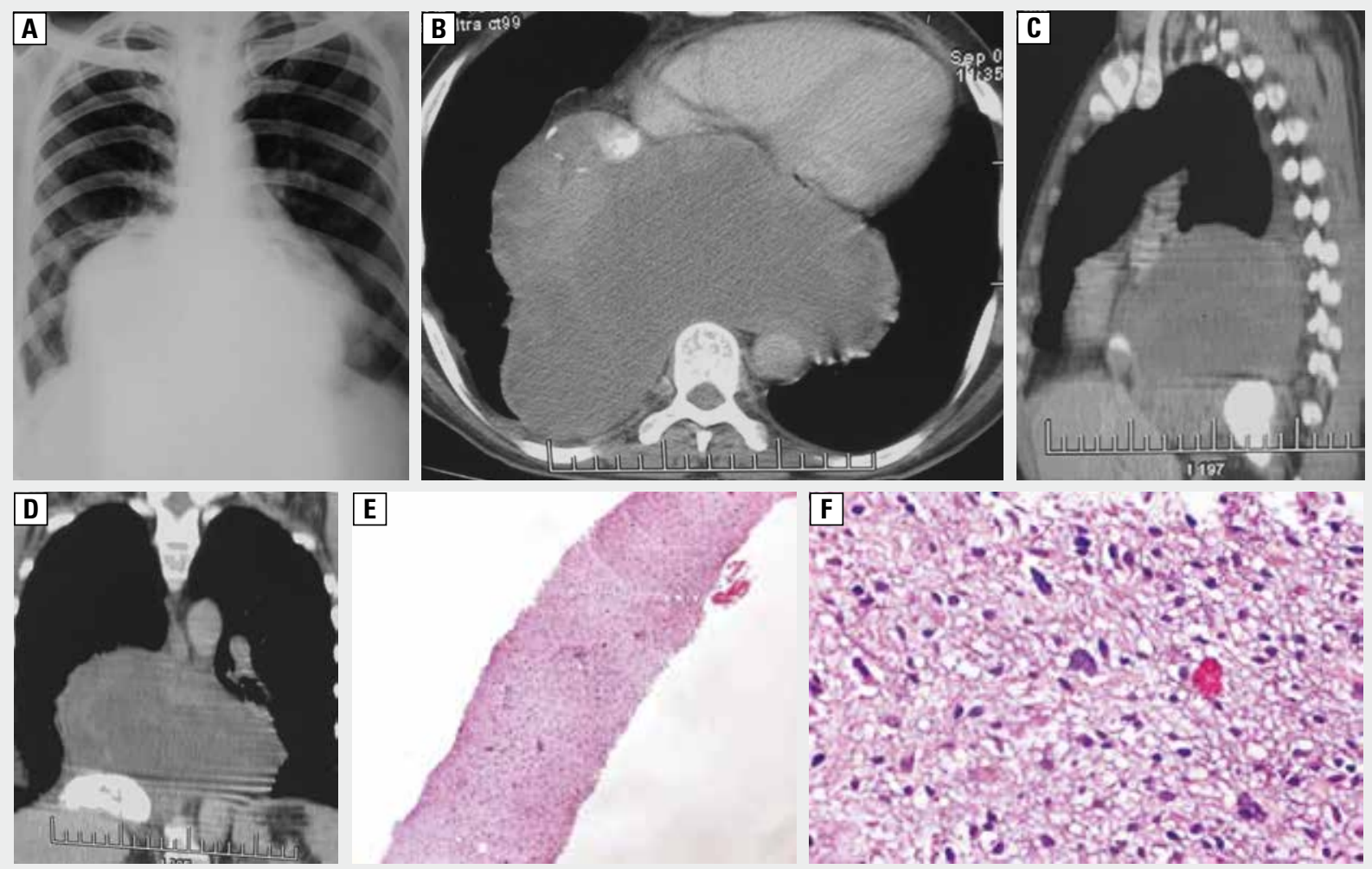

E]
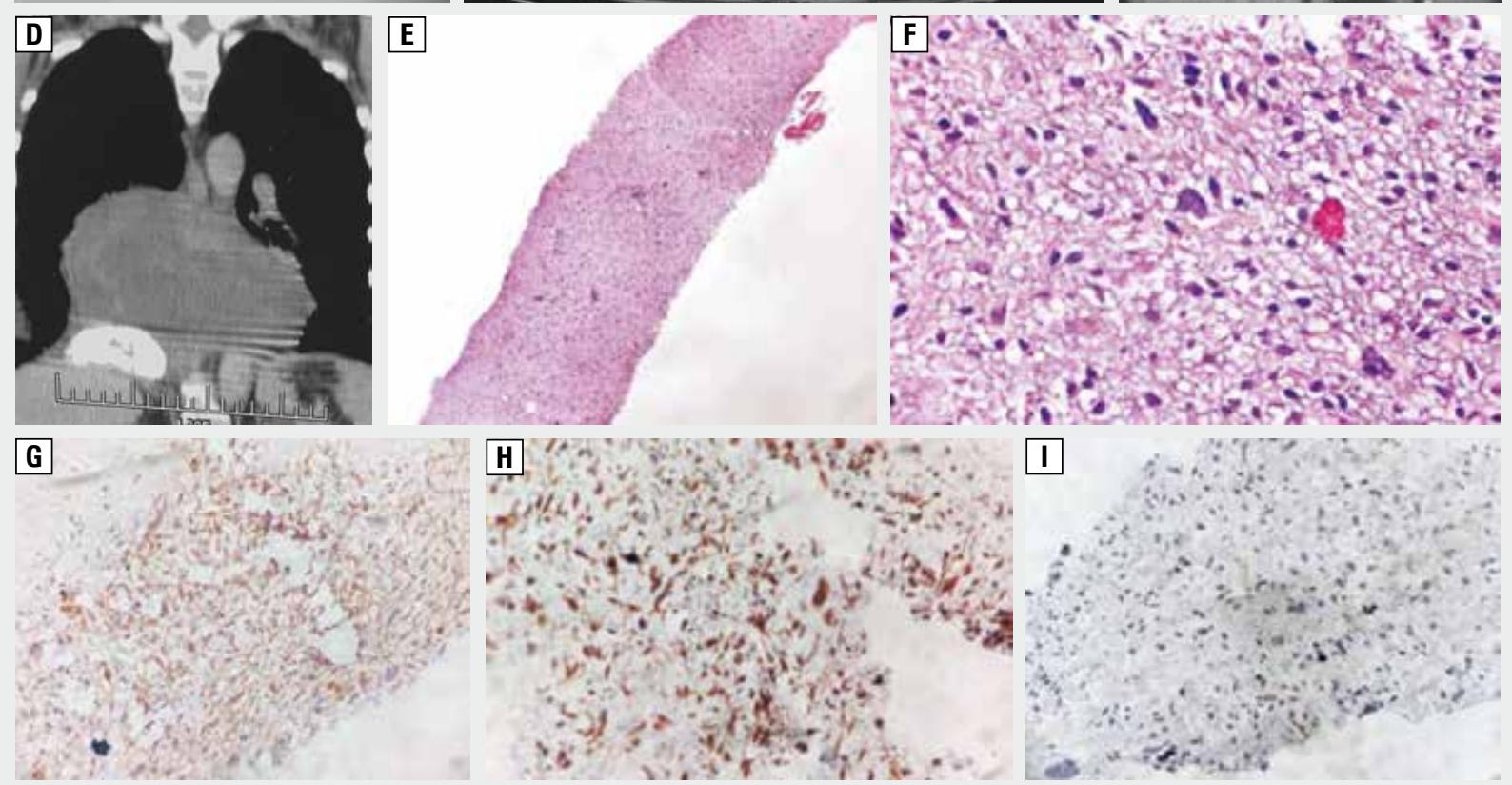

Figure 1. A. Frontal chest radiograph showing a mediastinal mass extending to the both sides of the midline, not silhouetting the cardiac outline. However, diaphragmatic outline is silhouetted, suggestive of likely posterior mediastinal origin; B. Axial CECT image shows heterogeneously enhancing solid posterior mediastinal mass extending to both sides of the midline showing areas of coarse calcification. Descending thoracic aorta is partially encased while the esophagus is not visualised, likely completely encased by the mass. No extension to neural foramen; $\mathbf{C}$ and D. Sagittal and coronal reformatted images reveal the vertically oriented fusiform configuration of the mass which is limited to the posterior mediastinum, not extending to the upper retroperitoneum. Note coarse calcification in its caudal aspect and mild right pleural effusion; E. Core biopsy from the soft tissue in low $(10 \mathrm{X})$ magnification mass showing tumour cells; $\mathbf{F}$. Higher magnification $(40 \mathrm{X})$ shows the tumour cells are arranged in diffuse sheets. These cells are pleomorphic, spindle-shaped to elongated, have eosinophilic cytoplasm and hyperchromatic irregular nuclei. Interspersed tumour giant cell can also be seen; $\mathbf{G}$. The tumour cells show vimentin positivity; $\mathbf{H}$. The tumour cells show CD34 positivity; I. The tumour cells are negative for S-100, SMA, Desmin, Calretinin, TTF-1, EMA, Bcl-2

\section{References:}

1. Feng L, Wang M, Yibulayin F, et al. Spindle cell sarcoma: a SEER population-based analysis. Sci Rep. 2018; 8(1): 5024, doi: 10.1038/ s41598-018-23145-4, indexed in Pubmed: 29568070.

2. Collini P, Sorensen PHB, Patel S, et al. Sarcomas with spindle cell morphology. Semin Oncol. 2009; 36(4): 324-337, doi: 10.1053/j.seminoncol.2009.06.007, indexed in Pubmed: 19664493.

3. Maki RG, Wathen JK, Patel SR, et al. Randomized phase II study of gemcitabine and docetaxel compared with gemcitabine alone in patients with metastatic soft tissue sarcomas: results of sarcoma alliance for research through collaboration study 002 [corrected]. J Clin Oncol. 2007; 25(19): 2755-2763, doi: 10.1200/JCO.2006.10.4117, indexed in Pubmed: 17602081. 